\title{
Interaction Between Adaptive Modulation and Scheduling for High-Rate Downlink Packet Transmission
}

\author{
Dong In Kim, Senior Member, IEEE
}

\begin{abstract}
This paper addresses adaptive modulation for downlink best effort packet data ("multiuser diversity") in a system employing high-order modulation and multicode (MC) directsequence code-division multiple-access formats. In particular, it analyzes the interaction between adaptive modulation and scheduling under the MC constraint that the number of MCs is relatively small (e.g., for relay-based downlink data transmission) and that all MCs to a given user have the same modulation. For this, two scheduling schemes are considered-two-best user and selective relative best user-that provide a balance between throughput and fairness, while accounting for MC and power constraints, high-order modulation, and channel coding for a fine rate granularity. To highlight the tradeoff, it compares the throughput and fairness of the conventional pure opportunistic, relative best, and round robin schemes with the two schemes and develops an analytical framework for the purpose.
\end{abstract}

Index Terms-Downlink, high-order modulation, multicode (MC) constraint, multiuser diversity, two-best (TB) user scheduling.

\section{INTRODUCTION}

A DAPTIVE modulation and transmission scheduling for high-rate data transmission over a direct-sequence codedivision multiple-access (DS-CDMA) downlink fading channel have received much attention recently [1], [2]. In combination, they provide a powerful tool to increase the average data rate, if delay is not a limiting factor and if a user can be scheduled for transmission when its instantaneous channel gain is near the peak. Nevertheless, there are some problems with schemes in which adaptive modulation is based only on selectable orders of modulation. For example, the granularity of the modulation family can lead to loss of efficiency, although channel coding renders a fine rate granularity [1]. Also, the maximum order of modulation is limited by the received signal-to-interference ratio (SIR) of users; since such modulations require a high received SIR, they are useful only for the best users, the ones that experience the most favorable channel condition.

Manuscript received November 20, 2004; revised November 28, 2005, June 6, 2006, and August 21, 2006. This work was supported in part by the Natural Sciences and Engineering Research Council (NSERC) of Canada. This paper was presented in part at the IEEE GLOBECOM '04 Conference, Dallas, TX, Nov. 2004. The review of this paper was coordinated by Prof. S.-L. Kim.

The author is with the School of Engineering Science, Simon Fraser University, Burnaby, BC V5A 1S6, Canada (e-mail: dikim@sfu.ca).

Color versions of one or more of the figures in this paper are available online at http://ieeexplore.ieee.org.

Digital Object Identifier 10.1109/TVT.2007.897222
However, DS-CDMA has alternative ways to support multiple and variable rates, such as controlling the spreading gain or using multiple orthogonal codes [3], [4]. This paper considers the latter scheme, namely, multicode (MC) DS-CDMA, where the spreading gain is fixed over a symbol time. We limit the number of MCs being used, because of fairness, bandwidth, and interference considerations in a cellular environment, ${ }^{1}$ and we require the modulation order selected by a given user to be the same over all MCs in order to simplify the receiver. Together, we term these restrictions the "multicode constraint." Although this is an improvement, compared with selecting modulation order only, there remains a limitation on the achievable rate, resulting from the MC constraint, that will affect the selection of efficient transmission scheduling schemes when the total transmitted power per cell is limited.

Turning now to transmission scheduling, we note that most schemes, to date, focus on how to assign the channel among users that experience time-varying fading in order to maximize total throughput, subject to certain constraints on fairness. The simplest pure opportunistic (PO) scheduling [6]-[8] selects the user with the best instantaneous channel. However, timevarying fading can be viewed as a mixture of short-term fading and long-term fadings (i.e., shadowing and path loss). Since PO scheduling is based on the instantaneous channel gain, it places users with poor long-term gains at a disadvantage for extended periods of time. A more satisfactory alternative is relative best (RB) scheduling [9], which mitigates such spatial differentiation among users by placing more weight on the short-term fading. It can be realized through the normalized channel gain, which is the ratio of the instantaneous channel gain to its local average (i.e., long-term fading).

This paper addresses the interaction between adaptive modulation and scheduling, subject to the MC constraint of real cellular DS-CDMA systems: a topic that has not yet been fully addressed. In particular, it analyzes the interaction of high-order modulation over MCs, transmission scheduling, and selectiverepeat (SR) error control with channel coding, ${ }^{2}$ and it considers a generalization of $\mathrm{PO}$ scheduling that provides a balance between throughput and fairness. In fact, scheduling more than one user at the same time can improve the rate granularity [2]

\footnotetext{
${ }^{1}$ Relay-based downlink data transmission [5] fits this situation well because of limited bandwidth and interference.

${ }^{2}$ The high-rate downlink transmission such as high-speed downlink packet access (HSDPA) [10] already implemented more sophisticated algorithms, but a theoretical framework developed here can largely be transferred to such applications.
} 


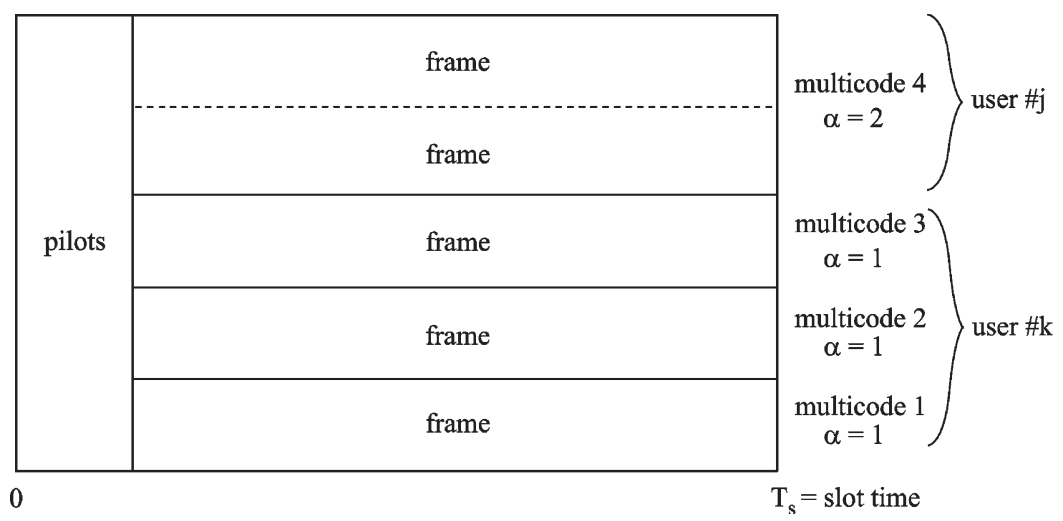

Fig. 1. Time-division multiplexing/multicode (TDM/MC)-based multiple frames downlink transmission.

under the MC constraint, which can also increase the fairness with a minimal loss in throughput. We refer to this scheme as two-best (TB) user scheduling in the sequel, and it can be generalized to schedule the third-best user and so on. Depending on the degree of MC constraint, there exists a compromise between PO and RB scheduling, in terms of both the throughput and fairness. This leads to the second generalization of RB, selective RB (SRB) scheduling, which combines the selection diversity of RB scheduling with the multiuser diversity of $\mathrm{PO}$ scheduling.

The rest of this paper is organized as follows. In Section II, the system model is given for the multirate and MC DS-CDMA downlink. The downlink SIR and resulting frame error rate (FER) are analyzed in Section III, where the target FER induces a target SIR, which, in turn, places constraints on the combined adaptive modulation and scheduling strategies. Section IV analyzes the sum-rate throughput for the scheduling schemes by partitioning the joint probability space conditioned on the maximum feasible rates. Numerical and simulation results are presented in Section $\mathrm{V}$, and concluding remarks are given in Section VI.

\section{SYSTEM MODEL}

An MC DS-CDMA is considered, which can transmit multiple bits using orthogonal parallel codes ("multicodes") in a synchronous downlink channel, all the MCs from a given base station (BS) being concatenated by a BS-specific PN sequence to distinguish the different transmissions. There is a finite and relatively small number of such MCs available to each BS. This paper is concerned with best effort packet data transmission, so that a user can be scheduled when its time-varying channel gain is at or near its peak. For this, a time-division transmission format is combined with the MC DS-CDMA format that leads to a time-division multiplexing (TDM)/MC/DS-CDMA system [1].

User data are carried in frames of fixed length. Several frames can be transmitted in parallel on different MCs. Also, depending on modulation order, more than one frame can be carried on each MC. To allow the mobile stations (MSs) to estimate the time-varying channel gain and SIR, the frames are preceded by pilot symbols. The combination of pilot symbols and parallel frames is termed a slot and is illustrated in Fig. 1.
To maintain integrity of the carried data, the system operates a hybrid SR protocol, in which an MS corrects a limited number of transmission errors. If the number of errors exceeds this limit (a frame error), the frame is not acknowledged, causing the BS to schedule a retransmission. A key design parameter affecting throughput is the target FER.

Different users experience independent channel conditions that combine short-term and long-term channel gains. For simplicity, and to highlight the role of multiuser diversity, the short-term channel gain is modeled as Rayleigh fading; thus, the instantaneous channel gain $\psi_{i}$ of the $i$ th user in a given cell has the density function

$$
f_{\psi_{i}}\left(x \mid \bar{\psi}_{i}\right)=\left(1 / \bar{\psi}_{i}\right) \exp \left(-x / \bar{\psi}_{i}\right), \quad x \geq 0
$$

with mean value $\bar{\psi}_{i}$. This paper has not addressed the use of multipath diversity at the MS, which would alter this density and, in general, reduce the effectiveness of multiuser diversity [9]. The long-term channel gain is characterized by the path loss and log-normal shadowing that vary with the user location in a cell; together, they determine $\bar{\psi}_{i}$. In this paper, the shortterm channel gain is assumed to be constant during a slot and to change from slot to slot, while the long-term gain remains constant over many slots, depending on the user mobility in a cellular environment. The channel fading is nominally flat in frequency; however, small amounts of delay spread have been accounted for in the next section.

In operation, each MS measures its downlink SIR and reports it to a target BS. Delayless error-free feedback is assumed in order to focus on the interplay between adaptive modulation and scheduling; however, the assumption will be revisited in a later publication. With knowledge of the SIRs of the MSs, the BS determines an optimum combination of $\mathrm{MC}$ and modulation formats for those MSs scheduled for transmission. Various scheduling policies are possible. The PO scheduling selects for any slot the single user with the largest SIR. Although it maximizes the total throughput, subject to a constraint on the total transmitted power, it causes a fairness problem, in the sense that a few users with relatively high long-term gains dominate access to the channel over extended periods. To mitigate the fairness problem, it is possible to normalize the instantaneous channel gains $\psi_{i}$ with their long-term gains $\bar{\psi}_{i}$; thus, all mobiles have normalized gains with the same exponential distribution. 
Selection on the basis of maximum normalized gain gives all mobiles the same short-term average access to the channel. We term this RB scheduling.

On the other hand, we may consider the policy that simultaneous transmission is allowed to the TB users, on the grounds that the second best of many users normally enjoys a very good channel, as well. With the policy, we trade a minimal loss in throughput for an increased fairness, which will be observed through analysis and simulations. When used in conjunction with absolute SIRs, we have a generalization of the PO policy, which is called TB scheduling. Furthermore, if we combine the absolute SIRs selectively with the normalized gains and SIRs, we obtain a variant of the RB policy, which is called SRB scheduling.

To describe the above scheduling policies in a well-defined manner, in the next section, a generalized SIR model is developed which allows selection of optimum modulation order, number of MCs, and error-correcting code rate to maximize throughput, subject to the MC constraint and constraints on the transmitted power and the FER. To facilitate the modeling of downlink SIR below, the TB scheduling for which the TB users are selected in a given slot is presumed.

\section{Modeling OF DownLink SIR}

A list of the key mathematical notations used in this paper is presented in Table I.

In this section, the downlink SIR is determined as a function of the controllable transmitter parameters: number of MCs, modulation level, and code rate assigned to the users. From the SIR, the symbol error rate (SER) is determined, then the FER. This gives the feasible parameter ranges that meet the target FER, providing a transmission model we take to the discussion of scheduling policies in the next sections.

Because of multilevel modulation, the transmit signal power changes at the symbol rate. An average power constraint is therefore more appropriate than a peak power constraint, and it is assumed that the peak power limitation of the amplifiers is relaxed enough to make this realistic. A given BS is assumed always to transmit at an assigned average total power of $\bar{P}_{B}$, regardless of the number of users and MCs and the modulation order.

Recognizing the limited complexity of an MS, the modulation order is limited to $\alpha=1,2,3$ for QPSK, 16-QAM, and 64-QAM, respectively, with the corresponding modulation levels $4^{\alpha}$. Further, the same modulation order is used on each of the MCs assigned to a given user. Then, the average total power transmitted by the tagged BS is derived as [11]

$$
\bar{P}_{B}=\frac{1}{3}\left[\left(4^{\alpha_{k}}-1\right) \phi_{k}+\left(4^{\alpha_{j}}-1\right) \phi_{j}\right] P_{b}
$$

where the indexes $k$ and $j$ denote the TB users, $\phi_{i}$ is the number of MCs $(i=k, j)$ with the code constraint $\phi_{k}+\phi_{j} \leq M$, and $P_{b}$ represents the power available to the mobile at basic rate (i.e., for $\alpha=1$, which corresponds to QPSK modulation) on a single code channel. Here, the rate allocation corresponding to the $i$ th user is determined by the parameters $\alpha_{i}$ and $\phi_{i}$, giving the transmission rate $m_{i}=\alpha_{i} \phi_{i}$ frames/slot, or $2 m_{i}$
TABLE I

LIST OF KEY NOTATIONS

\begin{tabular}{|c|c|}
\hline$\psi_{i}$ & Instantaneous channel gain of $i$ th user \\
\hline $\bar{\psi}_{i}$ & Long-term channel gain of $i$ th user \\
\hline$\delta$ & Path-loss factor \\
\hline$\sigma$ & Standard deviation of shadowing \\
\hline$\nu$ & In-cell orthogonality factor \\
\hline$\eta_{i}(n)$ & $\begin{array}{l}\text { Intercell interference factor } \\
\quad \text { of } i \text { th user from cell } n\end{array}$ \\
\hline$\mu_{i}$ & Effective interference factor of $i$ th user \\
\hline$K$ & No. active users per cell \\
\hline$N$ & Spreading gain per symbol \\
\hline$M$ & No. multicodes per cell \\
\hline$L$ & Radio frame length (bits) \\
\hline$\alpha_{i}(=1,2,3)$ & Modulation order allocated to $i$ th user \\
\hline$\phi_{i}(\leq M)$ & No. multicodes allocated to $i$ th user \\
\hline$m_{i}\left(=\alpha_{i} \phi_{i}\right)$ & Transmission rate allocated to $i$ th user \\
\hline$P_{b}$ & $\begin{array}{l}\text { Power allocation at basic rate } \\
\text { (per code channel) }\end{array}$ \\
\hline $\bar{P}_{B}$ & $\begin{array}{l}\text { Average power budget for BS } \\
\text { transmitters per cell }\end{array}$ \\
\hline$(\overline{S I R})_{o, i}$ & $\begin{array}{l}\text { Normalized SIR of } i \text { th user (per modulation } \\
\text { level per code channel) }\end{array}$ \\
\hline$(S I R)_{o, i}$ & $\begin{array}{l}\text { Average per-symbol SIR of } i \text { th user } \\
\text { (per code channel) }\end{array}$ \\
\hline$(S I R)_{o, i, p}$ & Pilot symbol SIR at basic rate \\
\hline$P_{e, i}$ & Symbol error rate (SER) of $i$ th user \\
\hline$P_{E, i}$ & Frame error rate (FER) of $i$ th user \\
\hline$t(=1,2, \ldots, 11)$ & Error-correction capability \\
\hline$r$ & Forward-error-correction code rate \\
\hline$P_{E}$ & Target FER \\
\hline$(\overline{S I R})_{o, \alpha}(t)$ & Normalized target SIR, given $(\alpha, t)$ \\
\hline $\bar{C}$ & Average sum-rate throughput \\
\hline $\mathcal{F}$ & Short-term fairness \\
\hline$R$ & Short-term fade rate (in cycle) \\
\hline
\end{tabular}

bits/symbol. There is no power control to the users other than their share of $\bar{P}_{B}$ determined implicitly by (2).

Now, to characterize the instantaneous channel gain $\psi_{i}$ as given in (1), the long-term gain is specified by the combined path loss and shadowing, where the mean $\bar{\psi}_{i}$ is defined by

$$
\bar{\psi}_{i}=r_{i}^{-\delta} \cdot 10^{\xi_{i} / 10} .
$$

It is assumed that, in (3), the signal power received at the $i$ th user from its BS is attenuated by the $\delta$ th power of the distance $r_{i}$ and log-normal shadowing $\left[\xi_{i}\right.$ is modeled as the normal distribution with zero mean and standard deviation $\sigma$ (decibel)].

Based on this channel gain model, and assuming that background noise is negligible compared with the composite of incell and intercell interferences, the normalized downlink SIR 
(per modulation level per code channel) for the $i$ th active user in a given cell is

$$
(\overline{\mathrm{SIR}})_{o, i}=\frac{\psi_{i} P_{b} N}{\psi_{i}(1-\nu) \bar{P}_{B}+\sum_{n \geq 1} \psi_{i, n} \bar{P}_{B, n}}
$$

where the average per-symbol SIR (per code channel) is defined by $(\mathrm{SIR})_{o, i}=(\overline{\mathrm{SIR}})_{o, i} \times\left[1 / 3\left(4^{\alpha_{i}}-1\right)\right], N$ is the spreading gain per symbol, and the index $n \geq 1$ indicates adjacent cells surrounding the cell under consideration. Note that the singlepath gain, as in (1), is assumed here for a tractable analysis of the multiuser diversity, but for an accurate estimation of the in-cell interference in various cellular environments (such as urban, rural area, and hilly terrain channels), a nonzero in-cell orthogonality factor $\nu \leq 1$ is introduced, which is caused by the loss of MC orthogonality due to multipath [12].

In this analysis, the steady-state system condition is assumed in which a total downlink power allocation is fully used to allow for the maximum possible rate(s) to a single user or the TB users. Hence, $\bar{P}_{B, n}=\bar{P}_{B}$ is assumed for all $n \geq 1$; otherwise, if $\bar{P}_{B, n}<\bar{P}_{B}$, it means that cell $n$ does not fully use the resource. Combining (2) and (4), the downlink SIR can be simplified to the following form:

$$
\begin{aligned}
(\overline{\mathrm{SIR}})_{o, i}= & 3 N\left[\left(4^{\alpha_{k}}-1\right) \phi_{k}+\left(4^{\alpha_{j}}-1\right) \phi_{j}\right]^{-1} \\
& \times\left[(1-\nu)+\sum_{n \geq 1} \eta_{i}(n)\right]^{-1}
\end{aligned}
$$

where $i=k, j$ (either one of the TB users), and the intercell interference factor $\eta_{i}(n)$ is defined by

$$
\eta_{i}(n) \triangleq \frac{\psi_{i, n}}{\psi_{i}}
$$

If the effective interference factor $\mu_{i}$ is further defined as

$$
\mu_{i} \triangleq\left[(1-\nu)+\sum_{n \geq 1} \eta_{i}(n)\right]
$$

then the downlink SIR in (5) can be expressed by the compact and tractable form

$$
(\overline{\mathrm{SIR}})_{o, i}=\frac{3 N}{\mu_{i}}\left[\left(4^{\alpha_{k}}-1\right) \phi_{k}+\left(4^{\alpha_{j}}-1\right) \phi_{j}\right]^{-1} .
$$

The interference factor $\mu_{i}(i=k, j)$ is measured by the MS using the pilot symbols in a downlink slot. They are inserted at the basic rate (i.e., QPSK with modulation order $\alpha=1$ ) on a single code channel. The downlink SIR for the pilot symbols, which is denoted by $(\mathrm{SIR})_{o, i, p}$, is measured as

$$
(\mathrm{SIR})_{o, i, p}=\frac{N}{\mu_{i}}
$$

and reported to the BS. Therefore, the TB $k$ th and $j$ th users' downlink SIRs can be estimated through the formula

$$
(\overline{\mathrm{SIR}})_{o, i}=(\mathrm{SIR})_{o, i, p} \times 3\left[\left(4^{\alpha_{k}}-1\right) \phi_{k}+\left(4^{\alpha_{j}}-1\right) \phi_{j}\right]^{-1}
$$

for $i=k$ and $j$, respectively.

The next analysis step is to relate the normalized SIR $(\overline{\mathrm{SIR}})_{o, i}$ of user $i$ to the user's SER $p_{e, i}$. For Gaussian-modeled interference, the SER can be approximated to [11]

$$
\begin{aligned}
p_{e, i}=4\left(1-2^{-\alpha_{i}}\right) Q & \left(\sqrt{(\overline{\mathrm{SIR}})_{o, i}}\right) \\
& -4\left(1-2^{-\alpha_{i}}\right)^{2} Q^{2}\left(\sqrt{(\overline{\mathrm{SIR}})_{o, i}}\right)
\end{aligned}
$$

where $Q(x)=1 / \sqrt{2 \pi} \int_{x}^{\infty} e^{-y^{2} / 2} d y$. With the modulation order $\alpha_{i}$, the bit error rate is well approximated by [11]

$$
p_{b, i} \cong \frac{1}{2 \alpha_{i}} p_{e, i}
$$

when the Gray encoding is employed.

Finally, the FER must be evaluated, resulting from the achieved downlink SIRs as given in (10). To find the FER for the $i$ th user with rate $m_{i}=\alpha_{i} \phi_{i}$, where $m_{i}$ frames are sent per slot, an SR type of hybrid automatic-repeat request (ARQ) error control scheme is considered, which attempts to correct $t$ errors and repeats the frame if that is not possible. For random errors, the FER is expressed as

$$
P_{E, i}=\sum_{e=t+1}^{L}\left(\begin{array}{l}
L \\
e
\end{array}\right) p_{b, i}^{e}\left(1-p_{b, i}\right)^{L-e}
$$

where $t$-bit error-correction capability is assumed for the frame of length $L$ bits and $\left(\begin{array}{l}k \\ l\end{array}\right) \triangleq k ! /(k-l) ! / l$ !. For a certain value of $t$, the code rate $r$ satisfies the Varsharmov-Gilbert lower bound [13] as

$$
r \geq 1-H\left(\frac{d_{\min }-2}{L}\right)
$$

where $H(x)=-x \log _{2} x-(1-x) \log _{2}(1-x)$ and the minimum Hamming distance $d_{\text {min }} \geq 2 t+1$.

Having determined the FER, we now work backwards to express the allowable combinations of modulation and coding parameters and pilot SIR that obtain a specified target FER $P_{E}$. For a certain value of $t$ (corresponding to $d_{\min }=2 t+1$ ), the required $d_{\min }$ and, hence, $r$ of a code with length $L$ equal to that of the frame can be determined (at least approximately) by (14). Also, if (11) and (12) are combined with (13), the target SIR is obtained, which is denoted by $(\overline{\mathrm{SIR}})_{o, \alpha}(t)$, associated with modulation order $\alpha$ and $t$-bit error-correction capability that obtains the target FER.

Table II shows the code rate $r$ for a certain value of $t$ according to the Varsharmov-Gilbert lower bound, while Table III presents the target SIRs to achieve the FER $P_{E}=0.1$, depending on $\alpha=1,2,3$ and $t=1,2, \ldots, 11$. 
TABLE II

Code Rate $r$ Versus Error-Correction CAPABility $t$ With Frame Length $L=128$

\begin{tabular}{||c|c|c|c|c|c|c|c|c|c|c|c||}
\hline$t$ & 1 & 2 & 3 & 4 & 5 & 6 & 7 & 8 & 9 & 10 & 11 \\
\hline$r$ & 0.934 & 0.840 & 0.762 & 0.694 & 0.633 & 0.577 & 0.526 & 0.479 & 0.435 & 0.394 & 0.356 \\
\hline
\end{tabular}

TABLE III

TARGET $(\overline{\mathrm{SIR}})_{o, \alpha}(t)$ BEING REQUIRED TO ACHIEVE THE FER $10^{-1}$

\begin{tabular}{||c|c|c|c|c|c|c|c|c|c|c|c||}
\hline & \multicolumn{10}{|c||}{$(\overline{S I R})_{o, \alpha}(t)(d B)$} \\
\cline { 2 - 14 }$\alpha$ & $t=1$ & $t=2$ & $t=3$ & $t=4$ & $t=5$ & $t=6$ & $t=7$ & $t=8$ & $t=9$ & $t=10$ & $t=11$ \\
\hline 1 & 8.42 & 7.52 & 6.86 & 6.31 & 5.83 & 5.40 & 5.01 & 4.64 & 4.30 & 3.96 & 3.65 \\
\hline 2 & 8.09 & 7.11 & 6.38 & 5.77 & 5.23 & 4.73 & 4.27 & 3.84 & 3.42 & 3.01 & 2.60 \\
\hline 3 & 7.77 & 6.72 & 5.92 & 5.24 & 4.62 & 4.06 & 3.52 & 2.99 & 2.48 & 1.97 & 1.45 \\
\hline
\end{tabular}

\section{A. TB Scheduling}

Given a set of the target SIRs $\left\{(\overline{\mathrm{SIR}})_{o, \alpha}(t)\right\}$ determined above (subject to the target FER), the procedure to allocate the optimum rates to the TB users is as follows.

1) The pilot SIRs $\left\{(\mathrm{SIR})_{o, i, p}\right\}$ are measured in a slot at the MSs and reported to the BS, where the TB users ( $k$ and $j$ ) are selected from $K$ active users as

$$
(\mathrm{SIR})_{o, k, p}=\max _{1 \leq i \leq K}\left\{(\mathrm{SIR})_{o, i, p}\right\}
$$

and

$$
(\mathrm{SIR})_{o, j, p}=\max _{\substack{1 \leq i \leq K \\ i \neq k}}\left\{(\mathrm{SIR})_{o, i, p}\right\}
$$

2) The optimum rates, which maximize the effective sum rate $\left(r_{k} \cdot \alpha_{k} \phi_{k}+r_{j} \cdot \alpha_{j} \phi_{j}\right)$, are allocated to the TB users subject to the target SIR constraints $(i=k, j)$ :

$$
\begin{aligned}
(\overline{\mathrm{SIR}})_{o, i} & =(\mathrm{SIR})_{o, i, p} \times 3\left[\left(4^{\alpha_{k}}-1\right) \phi_{k}+\left(4^{\alpha_{j}}-1\right) \phi_{j}\right]^{-1} \\
& \geq(\overline{\mathrm{SIR}})_{o, \alpha_{i}}\left(t_{i}\right)
\end{aligned}
$$

where the modulation order and $\mathrm{MCs}$, respectively, are constrained to $\alpha_{i}=1,2,3$ and $\phi_{k}+\phi_{j} \leq M$ with $\phi_{i} \geq 1$.

3) The power allocation per modulation level per code channel $P_{b}$ is determined by (2) subject to the average power constraint on $\bar{P}_{B}$.

Note that the PO scheduling corresponds to a special case of TB scheduling with $\alpha_{j}=\phi_{j}=0$.

\section{B. SRB Scheduling}

For a short-term fair access to the channel, we place more weight on the normalized gains $\left\{\psi_{i} / \bar{\psi}_{i}\right\}$ and SIRs as follows.

1) Using the measured pilot SIRs $\left\{(\mathrm{SIR})_{o, i, p}\right\}$ and their local time averages $\left\{\left\langle(\mathrm{SIR})_{o, i, p}\right\rangle\right\}$, the TB users $(k$ and $j$ ) are first selected as ${ }^{3}$

$$
k=\arg \max _{1 \leq i \leq K}\left\{\frac{\psi_{i}}{\bar{\psi}_{i}}\right\} \approx \arg \max _{1 \leq i \leq K}\left\{\frac{(\mathrm{SIR})_{o, i, p}}{\left\langle(\mathrm{SIR})_{o, i, p}\right\rangle}\right\}
$$

and $j$ in (17) for $1 \leq i \leq K$ and $i \neq k$.

\footnotetext{
${ }^{3}$ Note that, from (9) and (19), the in-cell interference $(1-\nu)$ can be kept small because of single-user transmission per slot, and $N \gg 1$; therefore, the approximation in (17) becomes accurate although not exact.
}

2) For a relative gain of multiuser diversity, the best user is then selected as the one with higher absolute SIR, namely, $k$ if $(\mathrm{SIR})_{o, k, p}>(\mathrm{SIR})_{o, j, p}$ and $j$ otherwise.

3) The optimum rates, which maximize the effective rate $r_{(1)} \cdot \alpha_{(1)} \phi_{(1)}$ for $(1)=k$ or $j$ in step 2), are allocated to the best user subject to the target SIR constraint

$$
\begin{aligned}
(\overline{\mathrm{SIR}})_{o,(1)} & =(\mathrm{SIR})_{o,(1), p} \times 3\left[\left(4^{\alpha_{(1)}}-1\right) \phi_{(1)}\right]^{-1} \\
& \geq(\overline{\mathrm{SIR}})_{o, \alpha_{(1)}}\left(t_{(1)}\right)
\end{aligned}
$$

where $\alpha_{(1)}=1,2,3$ and $1 \leq \phi_{(1)} \leq M$.

With RB scheduling, we choose only the best user $k$ in step 1) and allocate the optimum rates in (18) with $(1)=k$.

\section{AnAlysis of MaXimum Sum-Rate Throughrut}

For evaluation of the sum-rate throughput, the effective interference factor $\mu_{i}$ in (7) is first approximated to

$$
\mu_{i} \cong\left[(1-\nu)+\psi_{i}^{-1} \sum_{n \geq 1} \bar{\psi}_{i, n}\right]
$$

in multicell environments, i.e., $\sum_{n \geq 1} \psi_{i, n} \rightarrow \sum_{n \geq 1} \bar{\psi}_{i, n}$ because of the dominant distance-dependent loss, which will be validated by the simulation. Based on the approximation, the TB users $(i=k, j)$ as defined in (15) can equivalently be selected by

$$
k=\arg \max _{1 \leq i \leq K}\left\{\zeta_{i}\right\} \text { and } j=\arg \max _{\substack{1 \leq i \leq K \\ i \neq k}}\left\{\zeta_{i}\right\}
$$

where $\zeta_{i} \triangleq \psi_{i} /\left(\sum_{n \geq 1} \bar{\psi}_{i, n}\right)$, with the density function $f_{\zeta_{i}}\left(x \mid \bar{\zeta}_{i}\right)$, as given in (1), and $\bar{\zeta}_{i}=\bar{\psi}_{i} /\left(\sum_{n \geq 1} \bar{\psi}_{i, n}\right)$.

\section{A. TB Scheduling}

For the sum-rate throughput in TB scheduling, the joint density function of $\zeta_{(1)}$ and $\zeta_{(2)}$ associated with the TB users is 
derived as follows [14]:

$$
\begin{aligned}
f_{\zeta_{(1)}, \zeta(2)}\left(x, y \mid\left\{\bar{\zeta}_{i}\right\}\right)=\sum_{i=1}^{K} f_{\zeta_{i}}\left(x \mid \bar{\zeta}_{i}\right) & \sum_{\substack{l=1 \\
l \neq i \\
l \neq 1}}^{K} f_{\zeta_{l}}\left(y \mid \bar{\zeta}_{l}\right) \\
& \times \prod_{\substack{l^{\prime}=1 \\
l^{\prime} \neq i, l}}^{K} F_{\zeta_{l^{\prime}}}\left(y \mid \bar{\zeta}_{l^{\prime}}\right)
\end{aligned}
$$

where $x \geq y \geq 0$, and $F_{\zeta_{i}}\left(x \mid \bar{\zeta}_{i}\right)=1-\exp \left(-x / \bar{\zeta}_{i}\right)$ is the distribution function.

Define $C_{\mathrm{TB}}\left(r_{k}, r_{j}\right)$ by the maximum sum-rate throughput for TB scheduling subject to the SIR constraints, as given in (16), which is ${ }^{4}$

$$
\begin{aligned}
& C_{\mathrm{TB}}\left(r_{k}, r_{j}\right)=\max \left\{r_{k} \cdot \alpha_{k} \phi_{k}+r_{j} \cdot \alpha_{j} \phi_{j}\right\} \\
& \text { subject to } \zeta_{i} \geq \frac{\lambda_{i}\left(t_{i}\right)}{N \Omega^{-1}-\lambda_{i}\left(t_{i}\right)(1-\nu)} \quad \text { for } i=k, j
\end{aligned}
$$

where $\zeta_{k} \geq \zeta_{j} \geq 0, \lambda_{i}\left(t_{i}\right) \triangleq(\overline{\mathrm{SIR}})_{o, \alpha_{i}}\left(t_{i}\right)$ and $\Omega=\left[\left(4^{\alpha_{k}}-\right.\right.$ 1) $\left.\phi_{k}+\left(4^{\alpha_{j}}-1\right) \phi_{j}\right] / 3$.

In general, the optimal solution to the above constraints, which maximizes the sum-rate throughput, is a nonlinear function of the rate combinations $\left\{\alpha_{i}, \phi_{i} \mid i=k, j\right\}$ and the errorcorrection capabilities $\left(t_{k}, t_{j}\right)$, for which the joint probability space $(x, y)$ with $x \geq y \geq 0$ can be partitioned into a set of disjoint regions, conditioned on $\Omega$. For this, the region $R_{g}$ is defined in a descending order of $g$ such that

$$
R_{g} \triangleq\left\{(x, y) \mid x \geq x_{g}, y \geq y_{g}, x \geq y \geq 0\right\}-R_{g_{+}}
$$

where the boundary conditions $\left(x_{g}, y_{g}\right)$ are determined by

$$
\begin{aligned}
x_{g} & =\frac{\lambda_{k}\left(t_{k}\right)}{N g^{-1}-\lambda_{k}\left(t_{k}\right)(1-\nu)} \\
y_{g} & =\frac{\lambda_{j}\left(t_{j}\right)}{N g^{-1}-\lambda_{j}\left(t_{j}\right)(1-\nu)}
\end{aligned}
$$

for $x_{g} \geq y_{g} \geq 0, g=\Omega, g_{+}$is the smallest $\Omega_{+}$exceeding $g$, and the maximum value of $g$ is determined by choosing the maximum $\Omega^{*}$ s.t.

$$
\Omega^{*} \leq \min \left\{\frac{N}{\lambda_{k}\left(t_{k}\right)(1-\nu)}, \frac{N}{\lambda_{j}\left(t_{j}\right)(1-\nu)}, \max (\Omega)\right\}
$$

because of the feasibility condition $x \geq y \geq 0$.

Here, to ensure that the above set partitioning is optimal, in the sense that $C_{\mathrm{TB}}\left(r_{k}, r_{j}\right)$ in (22) can be achieved, the following conditions should be met.

1) The boundary conditions $\left(x_{g}, y_{g}\right)$ in $R_{g}$ should be determined to maximize $\left(r_{k} \cdot \alpha_{k} \phi_{k}+r_{j} \cdot \alpha_{j} \phi_{j}\right)$ for all possible rate combinations $\left\{\alpha_{i}, \phi_{i} \mid i=k, j\right\}$, subject to $g$.

\footnotetext{
${ }^{4}$ Here, the actual throughput is $\left(1-P_{E}\right) \cdot C_{\mathrm{TB}}$ that accounts for the reduction of transmission efficiency due to retransmission of the same frame by ARQ, given the target FER $P_{E}$.
}

2) A feasible set of $\left\{g \leq \Omega^{*}\right\}$ should be determined in a descending order of $g$ such that $R_{g}$ becomes a nonempty region in (23), and the maximum sum-rate throughput achieved in $\left\{R_{g}\right\}$ monotonously increases with $g$.

Using the set of partitioned regions $\left\{R_{g}\right\}$, the maximum sum-rate throughput is derived as

$$
\begin{aligned}
C_{\mathrm{TB}}\left(r_{k}, r_{j}\right)= & \sum_{g=1}^{\Omega^{*}} \max \left\{r_{k} \cdot \alpha_{k} \phi_{k}+r_{j} \cdot \alpha_{j} \phi_{j} \mid R_{g}\right\} \\
& \times \int_{(x, y) \in R_{g}} \int_{\zeta_{(1)}, \zeta_{(2)}}\left(x, y \mid\left\{\bar{\zeta}_{i}\right\}\right) d x d y .
\end{aligned}
$$

Since $x \geq y \geq 0$ in the joint probability space, it is necessary to maintain the target SIRs such that $\lambda_{k}\left(t_{k}\right) \geq \lambda_{j}\left(t_{j}\right)$ by properly selecting the modulation orders and the error-correction capabilities, which results in the maximum sum-rate throughput. Based on the observation, $C_{\mathrm{TB}}\left(r_{k}, r_{j}\right)$ in (26) can be evaluated in closed form in Appendix A as follows:

$$
\begin{aligned}
C_{\mathrm{TB}}\left(r_{k}, r_{j}\right)= & \sum_{g=1}^{\Omega_{-}^{*}} \max \left\{r_{k} \cdot \alpha_{k} \phi_{k}+r_{j} \cdot \alpha_{j} \phi_{j} \mid R_{g}\right\} \\
\times & {\left[\prod_{i=1}^{K} F_{i}\left(z_{g_{+}}\right)-\prod_{i=1}^{K} F_{i}\left(z_{g}\right)\right.} \\
& +\sum_{i=1}^{K}\left[1-F_{i}\left(z_{g_{+}}\right)\right] \prod_{l \neq i} F_{l}\left(y_{g_{+}}\right) \\
& \left.+\sum_{i=1}^{K}\left[1-F_{i}\left(z_{g}\right)\right] \prod_{l \neq i} F_{l}\left(y_{g}\right)\right] \\
& \times\left[\begin{array}{l}
1-\prod_{i=1}^{K} F_{i}\left(z_{\Omega^{*}}\right) \\
\end{array}\right. \\
& -\sum_{i=1}^{K}\left[1-\alpha_{k} \phi_{k}+r_{j} \cdot \alpha_{j} \phi_{j} \mid R_{\Omega^{*}}\right\} \\
& {\left.\left.\left[z_{\Omega^{*}}\right)\right] \prod_{l \neq i} F_{l}\left(y_{\Omega^{*}}\right)\right] }
\end{aligned}
$$

where $\Omega_{-}^{*}$ is the second largest value of $g, z_{g}=\max \left\{x_{g}, y_{g}\right\}$, and $F_{i}(x)=F_{\zeta_{i}}\left(x \mid \bar{\zeta}_{i}\right)$ has been assumed for notational simplicity.

Note that $F_{i}(x)$ in (27) is a function of $\bar{\zeta}_{i}$, i.e., $F_{i}(x)=1-$ $\exp \left(-x / \bar{\zeta}_{i}\right)$, where $\bar{\zeta}_{i}$ is given by

$$
\bar{\zeta}_{i}=\left[\sum_{n \geq 1} \frac{r_{i, n}^{-\delta} \cdot 10^{\xi_{i, n} / 10}}{r_{i}^{-\delta} \cdot 10^{\xi_{i} / 10}}\right]^{-1}
$$

Therefore, the average is taken with respect to $\left\{r_{i, n}, \xi_{i, n}\right\}$ for evaluation of the intercell interference in average sense, assuming $r_{i}^{-\delta} \cdot 10^{\xi_{i} / 10}>r_{i, n}^{-\delta} \cdot 10^{\xi_{i, n} / 10}$ for all $n \geq 1$ (i.e., the BS in 
the tagged cell is selected as the serving BS). In Appendix B, it can be shown that

$$
\begin{aligned}
\bar{F}_{i}(x) \triangleq & \mathbf{E}\left\{F_{i}(x) \mid \bar{\psi}_{i}>\bar{\psi}_{i, n}, \forall n \geq 1\right\} \\
\cong & 1-\int_{0}^{\frac{\sqrt{3}}{2}} \int_{0}^{\frac{u}{\sqrt{3}}} \prod_{0} \exp \left\{-x f(k, l) \times[g(u, v \mid k, l)]^{-\delta / 2}\right\} \\
& \left.\times\left(\frac{8}{\sqrt{3}}\right) d u d=2, j\right) \\
&
\end{aligned}
$$

where $\mathbf{E}$ denotes the expectation, $j=1,2, \ldots$ is any positive integer, $g(u, v \mid k, l) \triangleq\left((u+\sqrt{3} k / 2)^{2}+(v+3 l / 2)^{2} / u^{2}+v^{2}\right)$, and

$$
\begin{aligned}
f(k, l)= & \exp \left[(\sigma \ln 10 / 10)^{2}\right] \\
\times & \left\{1-Q\left[\frac{5 \delta}{\sqrt{2 \sigma^{2}}} \times \log _{10} g(u, v \mid k, l)\right.\right. \\
& \left.\left.-\sqrt{2 \sigma^{2}} \frac{\ln 10}{10}\right]\right\} .
\end{aligned}
$$

Using the average-sense intercell interference, the maximum sum-rate throughput in (27) can be estimated in average sense through $\left\{\bar{F}_{i}(x)\right\}$ instead of $\left\{F_{i}(x)\right\}$, which is denoted by $\bar{C}_{\mathrm{TB}}\left(r_{k}, r_{j}\right)$. It is to be noted that the average throughput can be maximized by jointly optimizing the code rates $\left(r_{k}, r_{j}\right)$ to be adaptive to the TB users' channel gains.

\section{B. PO Scheduling}

Notice that the PO scheduling is to allocate the maximum allowable rate to the best user in terms of $(\mathrm{SIR})_{o, k, p}$, as defined in (15) [or equivalently $\zeta_{k}$ in (20)], subject to the SIR constraint in (16) with $\alpha_{j}=\phi_{j}=0$. Hence, the maximum sumrate throughput can be expressed by

$$
\begin{aligned}
& C_{\mathrm{PO}}\left(r_{k}\right)=\max \left\{r_{k} \cdot \alpha_{k} \phi_{k}\right\} \\
& \text { subject to } \zeta_{k} \geq \frac{\lambda_{k}\left(t_{k}\right)}{N \Omega^{-1}-\lambda_{k}\left(t_{k}\right)(1-\nu)}
\end{aligned}
$$

where $\Omega=\left[\left(4^{\alpha_{k}}-1\right) \phi_{k}\right] / 3$, and the density function of $\zeta_{(1)}=$ $\max _{1 \leq i \leq K}\left\{\zeta_{i}\right\}$ is given by [14]

$$
f_{\zeta_{(1)}}\left(x \mid\left\{\bar{\zeta}_{i}\right\}\right)=\sum_{i=1}^{K} f_{\zeta_{i}}\left(x \mid \bar{\zeta}_{i}\right) \prod_{\substack{l=1 \\ l \neq i \\ l \neq i}}^{K} F_{\zeta_{l}}\left(x \mid \bar{\zeta}_{l}\right) .
$$

Similarly, by defining the disjoint regions $\left\{R_{g}\right\}$ as

$$
\begin{aligned}
R_{g} & \triangleq\left\{x \mid x \geq x_{g} \geq 0\right\}-R_{g_{+}} \\
\text {for } g & \leq \Omega^{*} \leq \min \left\{\frac{N}{\lambda_{k}\left(t_{k}\right)(1-\nu)}, \max (\Omega)\right\}
\end{aligned}
$$

the maximum sum-rate throughput for the PO scheduling can be derived as

$$
C_{\mathrm{PO}}\left(r_{k}\right)=\sum_{g=1}^{\Omega^{*}} \max \left\{r_{k} \cdot \alpha_{k} \phi_{k} \mid R_{g}\right\} \int_{x \in R_{g}} f_{\zeta_{(1)}}\left(x \mid\left\{\bar{\zeta}_{i}\right\}\right) d x
$$

and can be further evaluated in closed form, that is

$$
\begin{aligned}
C_{\mathrm{PO}}\left(r_{k}\right)= & \sum_{g=1}^{\Omega_{-}^{*}} \max \left\{r_{k} \cdot \alpha_{k} \phi_{k} \mid R_{g}\right\} \\
& \times\left[\prod_{i=1}^{K} F_{i}\left(x_{g_{+}}\right)-\prod_{i=1}^{K} F_{i}\left(x_{g}\right)\right] \\
& +\max \left\{r_{k} \cdot \alpha_{k} \phi_{k} \mid R_{\Omega^{*}}\right\} \\
& \times\left[1-\prod_{i=1}^{K} F_{i}\left(x_{\Omega^{*}}\right)\right]
\end{aligned}
$$

Then, the average-sense maximum sum-rate throughput $\bar{C}_{\mathrm{PO}}\left(r_{k}\right)$ is derived by replacing $\left\{F_{i}(x)\right\}$ in (35) with $\left\{\bar{F}_{i}(x)\right\}$ as given in (29).

\section{RB Scheduling}

When the RB scheduling is based on the short-term channel gains as $\left\{\psi_{i} / \bar{\psi}_{i}\right\}$, the density function of $\gamma_{i} \triangleq \psi_{i} / \bar{\psi}_{i}$ is given by $f_{\gamma_{i}}(x)=\exp (-x)$ for all $i$.

In (32), with $\zeta_{i}$ replaced by $\gamma_{i}$, the density function of $\gamma_{(1)}=$ $\max _{1 \leq i \leq K}\left\{\gamma_{i}\right\}$ is derived as

$$
f_{\gamma_{(1)}}(x)=K \exp (-x)[1-\exp (-x)]^{K-1}
$$

from which the density function of $\hat{\zeta}_{(1)}=\gamma_{(1)} \cdot \bar{\zeta}_{k}$ is shown to be

$$
f_{\hat{\zeta}_{(1)}}\left(x \mid \bar{\zeta}_{k}\right)=K f_{\zeta_{k}}\left(x \mid \bar{\zeta}_{k}\right)\left[F_{\zeta_{k}}\left(x \mid \bar{\zeta}_{k}\right)\right]^{K-1} .
$$

Combining this with (34) yields the maximum sum-rate throughput for the RB scheduling

$$
\begin{aligned}
C_{\mathrm{RB}}\left(r_{k}\right)= & \sum_{g=1}^{\Omega_{-}^{*}} \max \left\{r_{k} \cdot \alpha_{k} \phi_{k} \mid R_{g}\right\} \\
& \times\left[\left[F_{k}\left(x_{g_{+}}\right)\right]^{K}-\left[F_{k}\left(x_{g}\right)\right]^{K}\right] \\
& +\max \left\{r_{k} \cdot \alpha_{k} \phi_{k} \mid R_{\Omega^{*}}\right\} \\
& \times\left[1-\left[F_{k}\left(x_{\Omega^{*}}\right)\right]^{K}\right] .
\end{aligned}
$$

Since the expectation on $\left[F_{k}(x)\right]^{K}$ can be evaluated from (29) as

$$
\mathbf{E}\left\{\left[F_{k}(x)\right]^{K}\right\}=\sum_{n=0}^{K}(-1)^{n}\left(\begin{array}{l}
K \\
n
\end{array}\right)\left[1-\bar{F}_{k}(n x)\right]
$$


where $[1-\exp (-z)]^{K}=\sum_{n=0}^{K}(-1)^{n}\left(\begin{array}{l}K \\ n\end{array}\right) \exp (-n z)$ has been used; the average-sense maximum sum-rate throughput $\bar{C}_{\mathrm{RB}}\left(r_{k}\right)$ is found in (38) when $\left[F_{k}(x)\right]^{K}$ is replaced by (39).

\section{SRB Scheduling}

Similarly as in the RB scheduling, it can be shown that the joint density function of $\hat{\zeta}_{(1)}=\gamma_{(1)} \cdot \bar{\zeta}_{k}$ and $\hat{\zeta}_{(2)}=\gamma_{(2)} \cdot \bar{\zeta}_{j}$, where $\gamma_{(2)}=\max _{1 \leq i \neq(1) \leq K}\left\{\gamma_{i}\right\}$, is given by

$$
\begin{aligned}
f_{\hat{\zeta}_{(1)}, \hat{\zeta}_{(2)}}\left(x, y \mid \bar{\zeta}_{k}, \bar{\zeta}_{j}\right)=K(K-1) f_{\zeta_{k}}\left(x \mid \bar{\zeta}_{k}\right) f_{\zeta_{j}}\left(y \mid \bar{\zeta}_{j}\right) \\
\times\left[F_{\zeta_{j}}\left(y \mid \bar{\zeta}_{j}\right)\right]^{K-2}
\end{aligned}
$$

where $x \geq\left(\bar{\zeta}_{k} / \bar{\zeta}_{j}\right) y \geq 0$. With this joint density function, the distribution function of the maximum $\zeta_{(1)}=\max \left\{\hat{\zeta}_{(1)}, \hat{\zeta}_{(2)}\right\}$ can be derived in Appendix $\mathrm{C}$ as follows:

$$
F_{\zeta_{(1)}}\left(z \mid \bar{\zeta}_{k}, \bar{\zeta}_{j}\right)= \begin{cases}{\left[F_{\zeta_{k}}\left(z \mid \bar{\zeta}_{k}\right)\right]^{K},} & \text { for } \bar{\zeta}_{k} \geq \bar{\zeta}_{j} \\ K F_{\zeta_{k}}\left(z \mid \bar{\zeta}_{k}\right)\left[F_{\zeta_{j}}\left(z \mid \bar{\zeta}_{j}\right)\right]^{K-1} & \\ -(K-1)\left[F_{\zeta_{j}}\left(z \mid \bar{\zeta}_{j}\right)\right]^{K}, & \text { for } \bar{\zeta}_{k}<\bar{\zeta}_{j} .\end{cases}
$$

Therefore, the maximum sum-rate throughput for SRB scheduling has the expression

$$
C_{\mathrm{SRB}}\left(r_{i}\right)=\frac{1}{2} C_{\mathrm{SRB}}\left(r_{k} \mid \bar{\zeta}_{k} \geq \bar{\zeta}_{j}\right)+\frac{1}{2} C_{\mathrm{SRB}}\left(r_{i} \mid \bar{\zeta}_{k}<\bar{\zeta}_{j}\right)
$$

where $C_{\mathrm{SRB}}\left(r_{k} \mid \bar{\zeta}_{k} \geq \bar{\zeta}_{j}\right)=C_{\mathrm{RB}}\left(r_{k}\right)$ in (38), since the best user turns out to be the one with the highest normalized channel gain. Here, the factor $1 / 2$ is due to the independent and identically distributed long-term channel gains $\bar{\zeta}_{k}$ and $\bar{\zeta}_{j}$ associated with the TB users. Furthermore, $C_{\mathrm{SRB}}\left(r_{i} \mid \bar{\zeta}_{k}<\bar{\zeta}_{j}\right)$ ( $i=k$ or $j$ ) can be evaluated from (38) if $\left[F_{k}(x)\right]^{K}$ is replaced by $K F_{k}(x)\left[F_{j}(x)\right]^{K-1}-(K-1)\left[F_{j}(x)\right]^{K}$.

We note that an exact evaluation of the average-sense maximum sum-rate throughput requires those expectations $\left[F_{i}(x)\right]^{K}(i=k, j)$ and $F_{k}(x)\left[F_{j}(x)\right]^{K-1}$ conditioned on $\left\{\bar{\zeta}_{k} \geq \bar{\zeta}_{j}\right\}$ or $\left\{\bar{\zeta}_{k}<\bar{\zeta}_{j}\right\}$. It seems to be not tractable because of the dependence, and hence, we resort to the simulation to evaluate the throughput gain of SRB over RB in average sense.

\section{RESULTS}

The two generalized TB and SRB scheduling are compared with the conventional PO and RB scheduling, in terms of the sum-rate throughput and short-term fairness, while adaptive modulation is performed to yield the maximum throughput for the corresponding scheduling schemes. Here, the short-term fairness is measured through the formula [15]

$$
\mathcal{F}=\left[\sum_{k=1}^{K} \mathbf{E}\left\{m_{i}\right\}\right]^{2} /\left[K \sum_{k=1}^{K} \mathbf{E}^{2}\left\{m_{i}\right\}\right]
$$

where the value of this fairness ranges from $1 / K$ to 1.0 , with 1.0 corresponding to equal average downlink transmission rate for all mobiles in a tagged cell. The value of $1 / K$ corresponds to the extreme case where the average downlink transmission rate for all the mobiles (except one mobile) in the tagged cell is 0.0 .

For the comparison, it is assumed that the frame length is $L=128$ bits and spreading gain is $N=64$, because the error-correction capability $t \leq 11$ considered provides a proper range of code rates $r$ in Table II, resulting in a fine rate granularity with $L=128$ and due to the MC constraint $M \leq$ 22 with $N \geq M$. Here, the target FER $P_{E}=0.1$ is assumed for which a high modulation level is likely to be allocated with constrained $t \leq 11$ and $M \leq 22$. Note that the short-term fairness is measured over the time window of $10 \times R$ cycles, where $R$ denotes the short-term fade rate (in cycle) to the longterm channel gain. For instance, when $R=2$, the short-term gain changes twice faster than the long-term gain, given that the scheduling is performed every cycle. In-cell interference is assumed with the orthogonality factor $\nu=0.4,0.7,0.6$ for the urban, rural area, and hilly terrain channels, respectively, [12] while intercell interference is caused by the two-tier 18 adjacent cells of the target center cell with path-loss factor $\delta=4$ and standard deviation of shadowing $\sigma=8 \mathrm{~dB}$.

The average-sense maximum sum-rate throughputs $\bar{C}$ (optimized for $1 \leq t \leq 11$ ) for PO, RB, TB, SRB, and RR scheduling are compared in Figs. 2-4 for varying $M$ when in-cell interference varies as $\nu=0.4,0.6,0.7$, and $K=10$ users are active. Here, the short-term fade rate is assumed $R=20$ cycles over a fixed long-term channel gain. As the in-cell interference decreases with increasing $\nu$, we see that the TB outperforms the PO for increasing $M$, which is because the sum-rate becomes higher subject to the corresponding MC constraints. In fact, the degree of freedom in packing the rates with $\mathrm{TB}$ scheduling is higher than that of PO scheduling, even though a fine rate granularity is made by the coding. Meanwhile, the SRB increases the throughput compared to the RB because the former combines selection diversity (due to short-term fading) with part of multiuser diversity (gained by choosing the higher absolute SIR in the second stage). As anticipated, the RR performs worst, although it guarantees a fair access to the channel. We note that the numerical results obtained from the theoretical framework developed here fit well the simulation results, so that the framework can be used for a preliminary setup of key system parameters (e.g., code rates, modulation orders, and MCs).

To further observe the above sum-rate increases with TB and SRB scheduling, as compared to PO and RB scheduling, the long-term channel gain is controlled by the path-loss factor $\delta=3,4,5$ in Fig. 5, where the least in-cell interference is assumed under the rural area channel $(\nu=0.7)$, along with the MC constraint $M=16$. As the intercell interference decreases with increasing $\delta$, the TB offers increased gains over the PO because of the increase in sum rate. However, the multiuser diversity gained by the SRB is slightly reduced, resulting in reduced gains over the $\mathrm{RB}$, because the long-term gain becomes more favorable.

On the other hand, to see the effects of short-term fade rate on throughput and fairness, given that the scheduling is performed at each cycle of the short-term gain, they are plotted in Figs. 6 and 7 as a function of $R$ cycles when $K=10, M=16$, and 


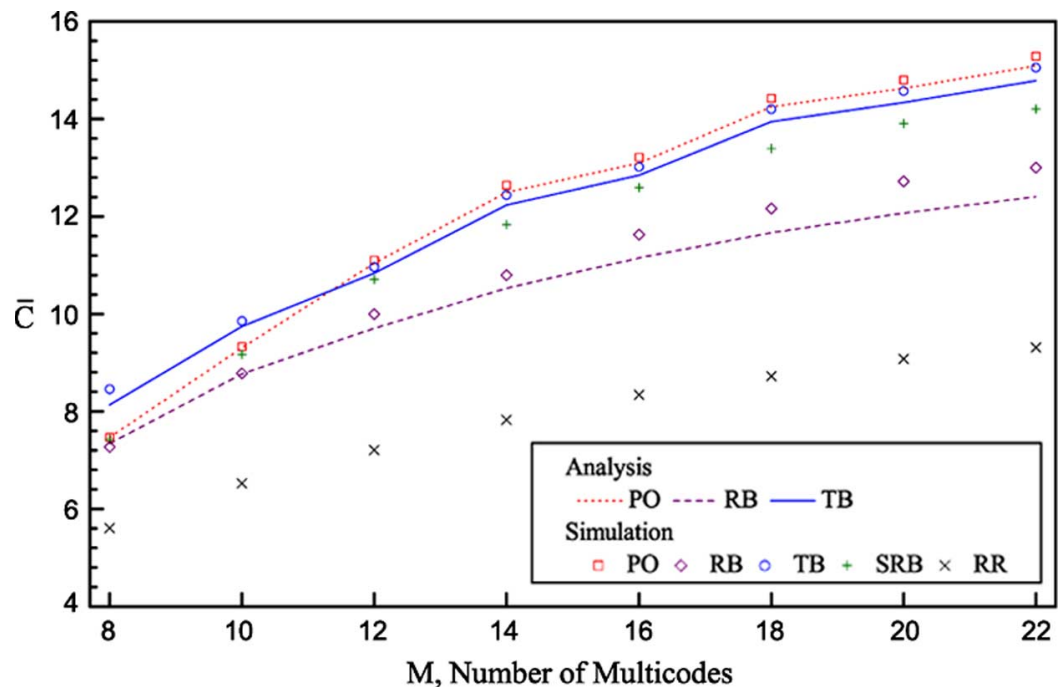

Fig. 2. Maximum throughput $\bar{C}$ versus $M$ when $K=10$ and $\nu=0.4$ (urban channel).

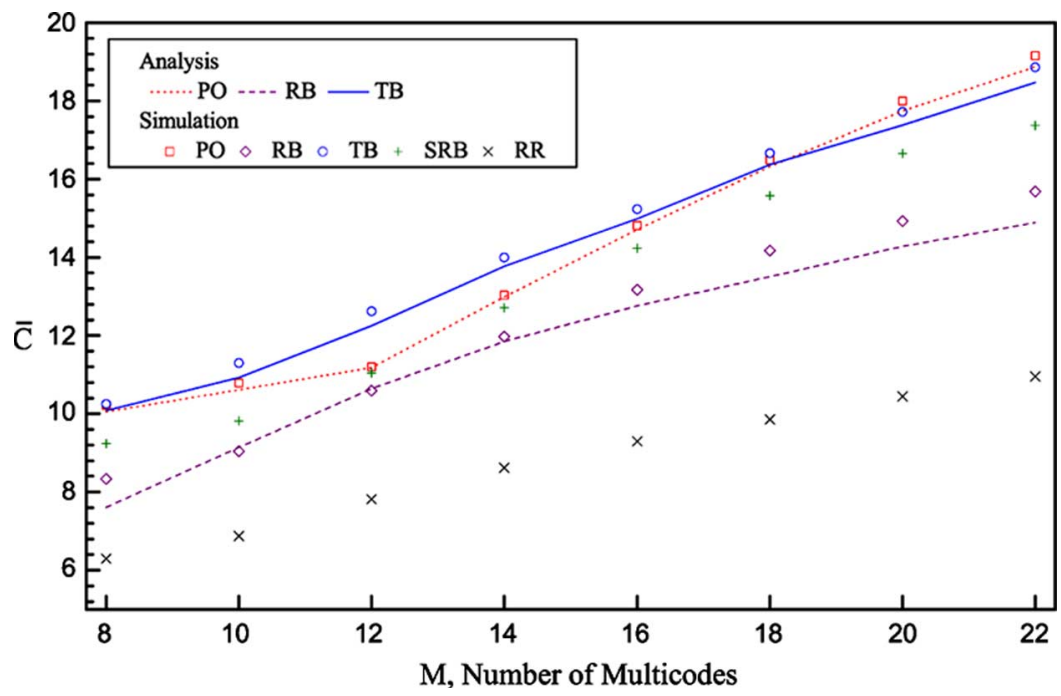

Fig. 3. Maximum throughput $\bar{C}$ versus $M$ when $K=10$ and $\nu=0.6$ (hilly terrain channel).

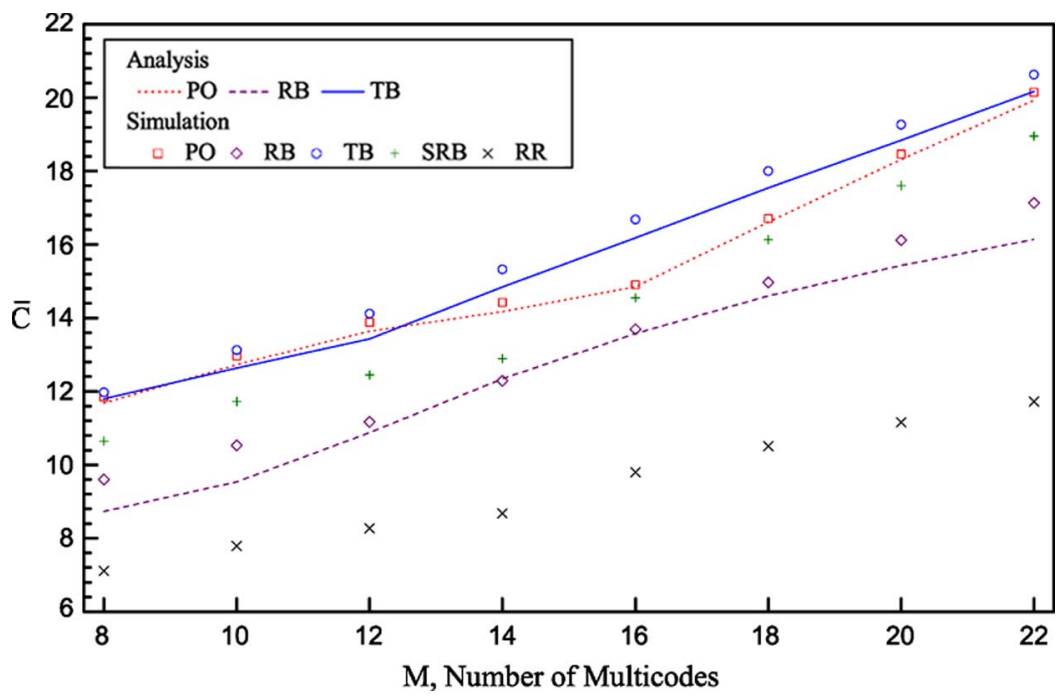

Fig. 4. Maximum throughput $\bar{C}$ versus $M$ when $K=10$ and $\nu=0.7$ (rural area channel). 


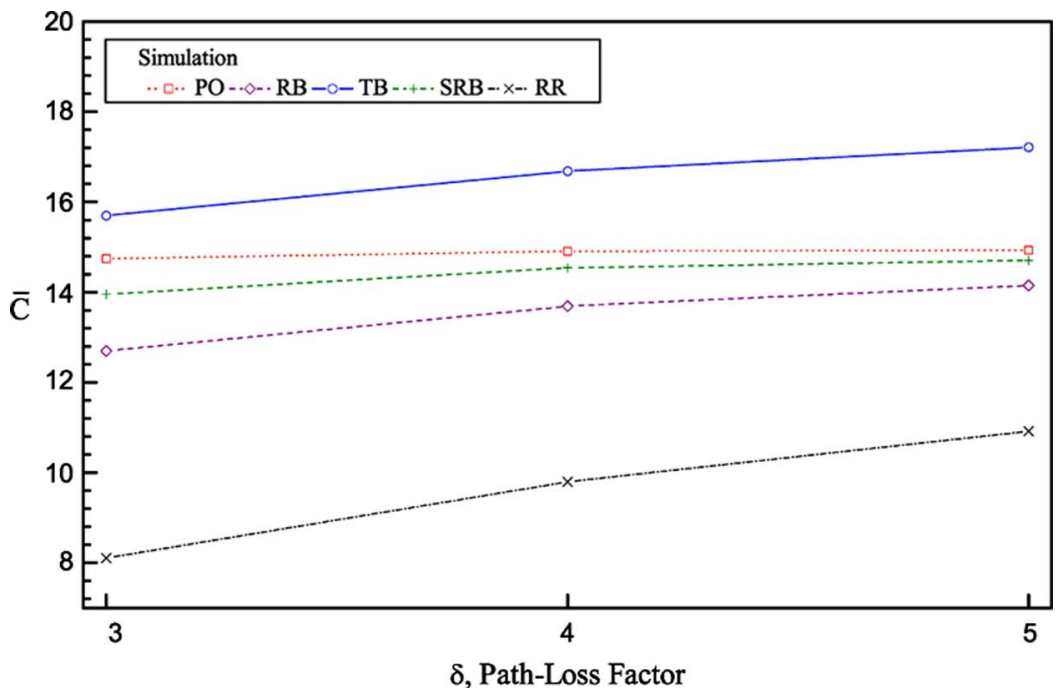

Fig. 5. Maximum throughput $\bar{C}$ versus $\delta$ when $K=10, M=16$, and $\nu=0.7$.

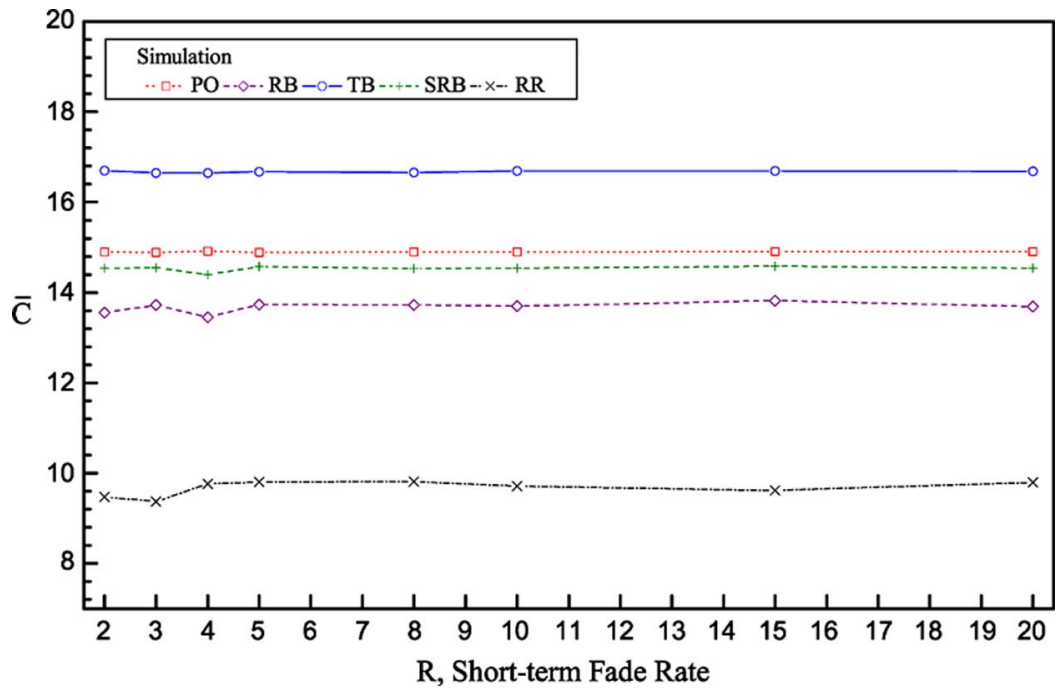

Fig. 6. Maximum throughput $\bar{C}$ versus $R$ when $K=10, M=16$, and $\nu=0.7$.

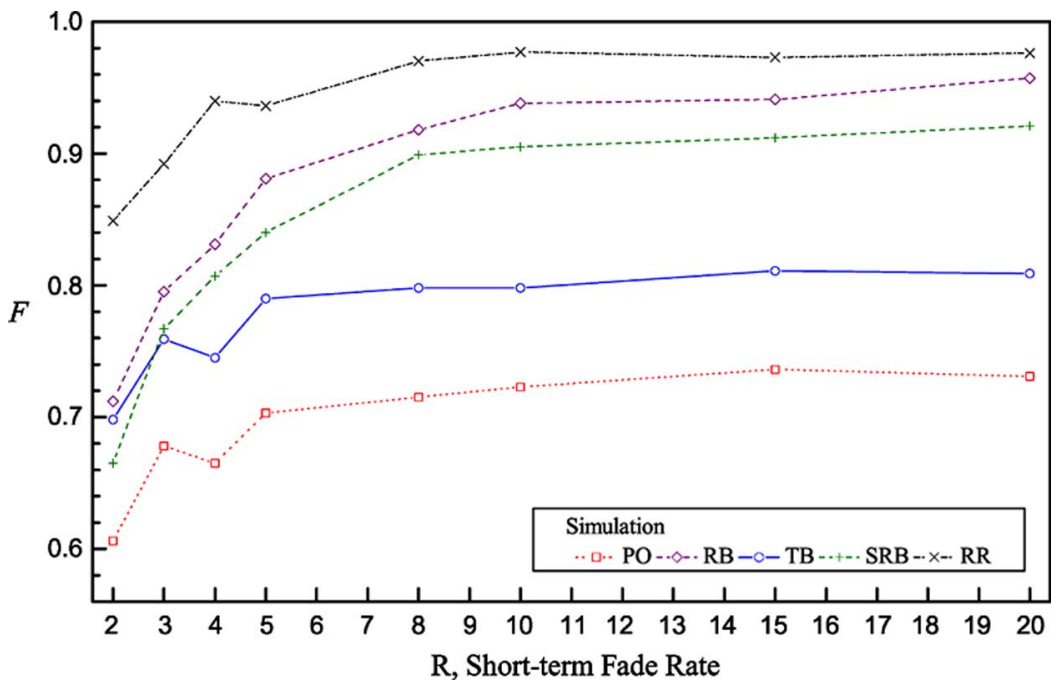

Fig. 7. Short-term fairness $\mathcal{F}$ versus $R$ when $K=10, M=16$, and $\nu=0.7$. 


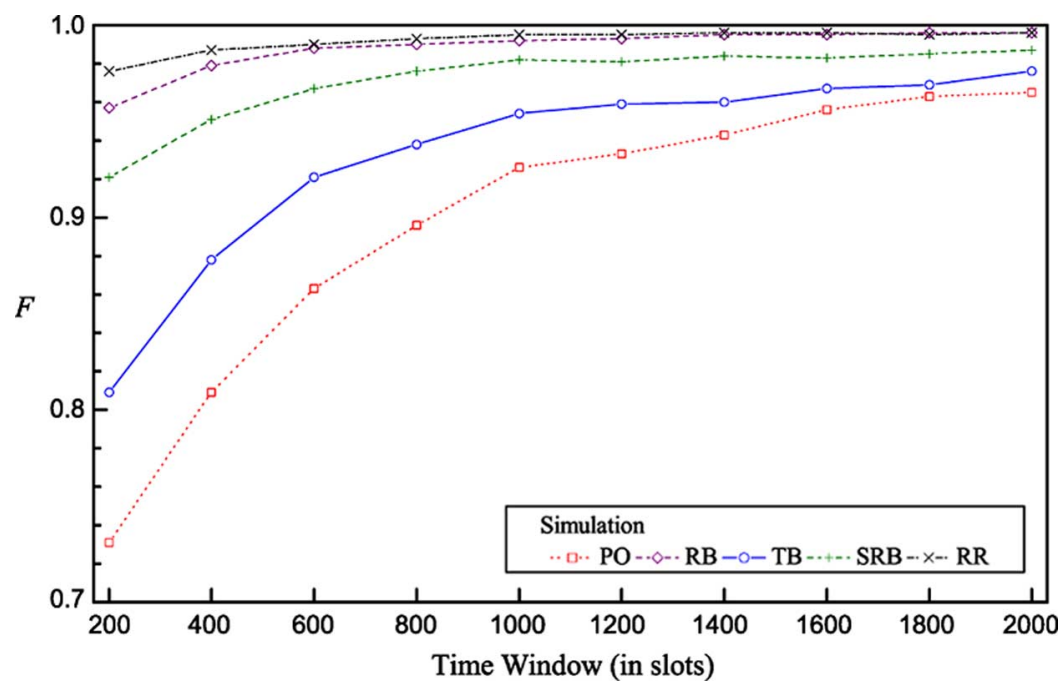

Fig. 8. Transient behavior of short-term fairness $\mathcal{F}$ versus window when $K=10, M=16$, and $\nu=0.7$.

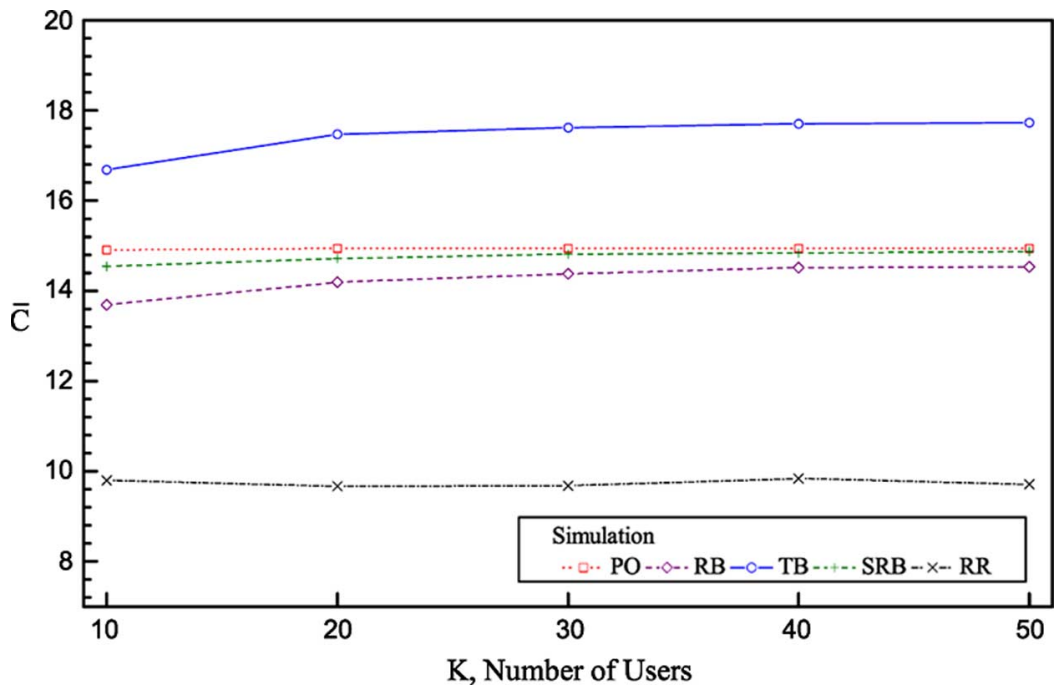

Fig. 9. Maximum throughput $\bar{C}$ versus $K$ when $K=10, M=16$, and $\nu=0.7$.

$\nu=0.7$. First, the throughput remains almost unchanged as $R$ varies from 2 to 20 cycles, because the best user out of $K=10$ active users picks up the most favorable channel gain for the $\mathrm{PO}$ and $\mathrm{TB}$, even with varying $R$ (i.e., sufficient multiuser diversity exists). The selection diversity with RB and SRB fluctuates initially but enters a stable region after $R=5$ cycles over a fixed long-term gain. However, the short-term fairness is sensitive to the short-term fade rate since scheduling is adaptive to the fade rate but reaches the steady-state fairness after $R=10$ cycles. As anticipated, the TB improves the short-term fairness compared to the $\mathrm{PO}$ while increasing the flexibility of packing the higher sum-rate under the MC constraint. Also, it is noticeable that the SRB provides a balance between throughput and fairness when compared to the RB.

Next, to look into the transient behavior of short-term fairness, the fairness $\mathcal{F}$ is plotted as a function of the time window in Fig. 8 when $K=10, M=16, R=20$, and $\nu=0.7$. Here, we assume that one cycle of the short-term gain occurs in a slot, and if $R=10$, then the short-term gain changes every other slot. First, we observe that the RB guarantees the maximum fairness as the RR does, if the window is sufficiently large (i.e., 1400 slots). Second, the PO, TB, and SRB cannot achieve the maximum fairness unlike the RB and RR, even if the window is sufficiently large (i.e., 2000 slots). To overcome the longterm unfairness, the TB and SRB may adopt Liu's opportunistic algorithm [8] rather than the $\mathrm{PO}$ where an offset is added to the performance value (SIR here) to meet the minimum fairness requirement. Third, the TB offers a certain gain over the PO in terms of the short-term fairness when the window of less than 1000 slots is used to meet a tight delay requirement.

Finally, the gain offered by multiuser diversity is investigated as $K$ increases in Fig. 9 when $M=16, R=20$, and $\nu=0.7$. It is seen that the gain is not so significant, but the TB benefits the most by the multiuser diversity, as anticipated. Hence, the TB will perform better when $K$ becomes relatively large, which is often observed in hotspot cells. 


\section{CONCLUSION}

This paper has investigated the interaction between adaptive modulation and scheduling under the constraints on modulation order, MCs, and transmit power, and observed that the sum rate can be increased by scheduling the TB users with highest channel gains. Also, the SRB scheduling was shown to provide a balance between throughput and fairness by combining selection diversity with part of multiuser diversity. The TB and SRB scheduling could be generalized by incorporating Liu's (rather than pure) opportunistic algorithm to achieve the longterm fairness, like the RB and RR scheduling. Finally, the analytical framework developed here will be used to optimize key system parameters such as modulation and MC formats with coding, depending on the scheduling algorithms, and channel and interference conditions in relay-based downlink data transmission.

\section{APPENDIX A \\ EVALUATION OF $C_{\mathrm{TB}}\left(r_{k}, r_{j}\right)$ IN (27)}

The double integration over the region $R_{g}$ in (26) can be performed for the two cases such as $\left\{R_{g} \mid 1 \leq g \leq \Omega_{-}^{*}\right\}$ and $R_{\Omega^{*}}$, respectively. First, for the region $R_{\Omega^{*}}$, it can be evaluated as

$$
\begin{aligned}
\int_{(x, y) \in R_{\Omega^{*}}} \int_{\zeta_{(1)}, \zeta_{(2)}} & \left(x, y \mid\left\{\bar{\zeta}_{i}\right\}\right) d x d y \\
& =\int_{x_{\Omega^{*}}} \int_{y_{\Omega^{*}}}^{x} f_{\zeta_{(1)}, \zeta_{(2)}}\left(x, y \mid\left\{\bar{\zeta}_{i}\right\}\right) d x d y
\end{aligned}
$$

if $x_{\Omega^{*}} \geq y_{\Omega^{*}}$; otherwise, the $x$-integration is evaluated by replacing $x_{\Omega^{*}}$ with $y_{\Omega^{*}}$ in (44), while the $y$-integration remains the same. Next, we obtain that for the region $R_{g}, 1 \leq g \leq \Omega_{-}^{*}$

$$
\begin{aligned}
\int_{(x, y) \in R_{\Omega^{*}}} & f_{\zeta_{(1)}, \zeta_{(2)}}\left(x, y \mid\left\{\bar{\zeta}_{i}\right\}\right) d x d y \\
= & \int_{x_{g}} \int_{y_{g}}^{x_{g_{+}}} f_{\zeta_{(1)}, \zeta_{(2)}}\left(x, y \mid\left\{\bar{\zeta}_{i}\right\}\right) d x d y \\
& +\int_{x_{g_{+}}}^{\infty} \int_{y_{g}}^{y_{g_{+}}} f_{\zeta_{(1)}, \zeta_{(2)}}\left(x, y \mid\left\{\bar{\zeta}_{i}\right\}\right) d x d y
\end{aligned}
$$

if $x_{g} \geq y_{g}$ and $x_{g_{+}} \geq y_{g_{+}}$, and if $x_{g} \geq y_{g}$ but $x_{g_{+}}<y_{g_{+}}$, the $x$-integration is evaluated by replacing $x_{g_{+}}$with $y_{g_{+}}$in (45). If both $x_{g}<y_{g}$ and $x_{g_{+}}<y_{g_{+}}$, it is evaluated by replacing $\left(x_{g}, x_{g_{+}}\right)$with $\left(y_{g}, y_{g_{+}}\right)$, respectively. Then, if we combine (44) and (45) with (26) for $g=1,2, \ldots, \Omega^{*}, C_{\mathrm{TB}}\left(r_{k}, r_{j}\right)$ is evaluated as (27).

\section{APPENDIX B \\ EVALUATION OF $\bar{F}_{i}(x)$ IN (29)}

Let $\theta_{i, n}=\xi_{i, n}-\xi_{i}$ in (28) and the coordinates $(u, v)$ denote a location of the $i$ th user in the tagged cell, and then ${ }^{5}$

$$
\begin{aligned}
& \mathbf{E}\left\{F_{i}(x) \mid \bar{\psi}_{i}>\bar{\psi}_{i, n}, \forall n \geq 1\right\} \\
& \cong 1-\mathbf{E}_{(u, v)} \\
& \quad \times\left\{\mathbf { E } _ { \theta _ { i , ( k , l ) } } \left\{\prod _ { \substack { ( \pm k , \pm l ) \\
k + l = 2 j } } \operatorname { e x p } \left\{-x[g(u, v \mid k, l)]^{-\delta / 2}\right.\right.\right. \\
& \left.\left.\left.\times 10^{\theta_{i,(k, l)} / 10} 1_{\theta_{i,(k, l)}}\right\}\right\}\right\}
\end{aligned}
$$

where $(k, l) \equiv n \geq 1$, and $\mathbf{1}_{\theta}=1$ if $\theta<5 \delta \log _{10} g(u, v \mid k, l)$. Using Jensen's inequality [16], where $\mathbf{E}\{f[g(\theta)]\} \cong$ $f[\mathbf{E}\{g(\theta)\}]$ for the functions $f(\cdot)$ and $g(\cdot)$, the above expectation can be further approximated to

$$
\begin{aligned}
& \mathbf{E}\left\{F_{i}(x) \mid \bar{\psi}_{i}>\bar{\psi}_{i, n}, \forall n \geq 1\right\} \\
& \cong 1-\mathbf{E}_{(u, v)}\left\{\prod _ { \substack { ( \begin{array} { c } 
{ \pm k , \pm l \\
k + l = 2 j }
\end{array} ) \\
} } \operatorname { e x p } \left\{-x[g(u, v \mid k, l)]^{-\delta / 2}\right.\right. \\
& \left.\left.\quad \times \mathbf{E}_{\theta_{i,(k, l)}}\left\{10^{\theta_{i,(k, l)} / 10} 1_{\theta_{i,(k, l)}}\right\}\right\}\right\} .
\end{aligned}
$$

Following the approaches in [15] and [17], we obtain the approximation to $\bar{F}_{i}(x)$ in (29).

\section{APPENDIX C \\ DERIVATION OF $F_{\zeta_{(1)}}\left(z \mid \bar{\zeta}_{k}, \bar{\zeta}_{j}\right)$ IN (41)}

Since $\hat{\zeta}_{(1)} / \bar{\zeta}_{k}=\gamma_{(1)} \geq \gamma_{(2)}=\hat{\zeta}_{(2)} / \bar{\zeta}_{j} \geq 0$, we know that $\hat{\zeta}_{(2)} \leq \kappa \cdot \hat{\zeta}_{(1)}$, where $\kappa=\bar{\zeta}_{j} / \bar{\zeta}_{k}$. Hence, if $\kappa \leq 1$, the joint density function can be formulated as

$$
F_{\zeta_{(1)}}\left(z \mid \bar{\zeta}_{k}, \bar{\zeta}_{j}\right)=\int_{0}^{z} \int_{0}^{\kappa x} f_{\hat{\zeta}_{(1)}, \hat{\zeta}_{(2)}}\left(x, y \mid \bar{\zeta}_{k}, \bar{\zeta}_{j}\right) d x d y .
$$

Otherwise, it can be expressed by

$$
\begin{aligned}
F_{\zeta_{(1)}}\left(z \mid \bar{\zeta}_{k}, \bar{\zeta}_{j}\right)= & \int_{0}^{z / \kappa} \int_{0}^{\kappa x} f_{\hat{\zeta}_{(1)}, \hat{\zeta}_{(2)}}\left(x, y \mid \bar{\zeta}_{k}, \bar{\zeta}_{j}\right) d x d y \\
& +\int_{z / \kappa}^{z} \int_{0}^{z} f_{\hat{\zeta}_{(1)}, \hat{\zeta}_{(2)}}\left(x, y \mid \bar{\zeta}_{k}, \bar{\zeta}_{j}\right) d x d y .
\end{aligned}
$$

Therefore, if we combine the two cases above, $F_{\zeta_{(1)}}\left(z \mid \bar{\zeta}_{k}, \bar{\zeta}_{j}\right)$ is derived as (41).

\footnotetext{
${ }^{5}$ Here, the interference is somewhat underestimated due to decreased $\eta_{i}(n)$ in (6) compared to the case of soft handoff.
} 


\section{ACKNOWLEDGMENT}

The author would like to thank J. K. Cavers for stimulating this research and helpful discussions.

\section{REFERENCES}

[1] P. Bender et al., "CDMA/HDR: A bandwidth-efficient high-speed wireless data service for nomadic users," IEEE Commun. Mag., vol. 38, no. 7, pp. 70-77, Jul. 2000.

[2] S. Parkvall et al., "The high speed packet data evolution of WCDMA," in Proc. IEEE VTC-Spring, 2001, vol. 3, pp. 2287-2291.

[3] F. Adachi, M. Sawahashi, and H. Suda, "Wideband DS-CDMA for next-generation mobile communications system," IEEE Commun. Mag., vol. 36, no. 9 , pp. 56-69, Sep. 1998.

[4] E. Dahlman et al., "UMTS/IMT-2000 based on wideband CDMA," IEEE Commun. Mag., vol. 36, no. 9, pp. 70-80, Sep. 1998.

[5] R. Pabst et al., "Relay-based deployment concepts for wireless and mobile broadband radio," IEEE Commun. Mag., vol. 42, no. 9, pp. 80-89, Sep. 2004.

[6] X. Liu, E. K. P. Chong, and N. B. Shroff, "Opportunistic transmission scheduling with resource-sharing constraints in wireless networks," IEEE J. Sel. Areas Commun., vol. 19, no. 10, pp. 2053-2064, Oct. 2001.

[7] P. Viswanath, D. N. C. Tse, and R. Laroia, "Opportunistic beamforming using dumb antennas," IEEE Trans. Inf. Theory, vol. 48, no. 6, pp. 12771294, Jun. 2002.

[8] X. Liu, E. K. P. Chong, and N. B. Shroff, "A framework for opportunistic scheduling in wireless networks," Comput. Netw., vol. 41, no. 4, pp. 451474, Mar. 2003.

[9] F. Berggren and R. Jantti, “Asymptotically fair transmission scheduling over fading channel," IEEE Trans. Wireless Commun., vol. 3, no. 1, pp. 326-336, Jan. 2004.

[10] S. Parkvall et al., "Evolving 3G mobile systems: Broadband and broadcast services in WCDMA," IEEE Commun. Mag., vol. 44, no. 2, pp. 68-74, Feb. 2006.

[11] J. G. Proakis, Digital Communications, 4th ed. New York: McGrawHill, 2001.

[12] N. B. Mehta et al., "Analysis and results for the orthogonality factor in WCDMA downlinks," IEEE Trans. Wireless Commun., vol. 2, no. 6, pp. 1138-1149, Nov. 2003.

[13] W. W. Peterson and E. J. Weldon, Error Correcting Codes. Cambridge, MA: MIT Press, 1972.
[14] N. Kong and L. B. Milstein, "Average SNR of a generalized diversity selection combining scheme," IEEE Commun. Let., vol. 3, no. 3, pp. 5759, Mar. 1999.

[15] D. I. Kim, E. Hossain, and V. K. Bhargava, "Downlink joint rate and power allocation in cellular multi-rate WCDMA systems," IEEE Trans. Wireless Commun., vol. 2, no. 1, pp. 69-81, Jan. 2003.

[16] E. A. Geraniotis, "Throughput and packet error probability tradeoffs in cellular direct-sequence and hybrid spread-spectrum networks," in Proc. IEEE ICC, Jun. 1987, pp. 40.4.1-40.4.6.

[17] K. S. Gilhousen et al., "On the capacity of a cellular CDMA system," IEEE Trans. Veh. Technol., vol. 4, no. 2, pp. 303-312, May 1991.

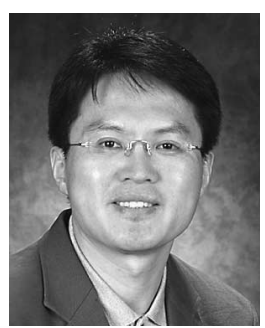

Dong In Kim (S'89-M'91-SM'02) received the B.S. and M.S. degrees in electronics engineering from Seoul National University, Seoul, Korea, in 1980 and 1984, respectively, and the M.S. and Ph.D. degrees in electrical engineering from the University of Southern California (USC), Los Angeles, in 1987 and 1990, respectively.

From 1984 to 1985 , he was with Korea Telecom Research Center, Seoul, as a Researcher. During 1986-1988, he was a Korean Government Graduate Fellow with the Department of Electrical Engineering, USC. From 1991 to 2002, he was with the University of Seoul, leading the Wireless Communications Research Group. Since 2002, he has been with Simon Fraser University, Burnaby, BC, Canada, where he is a Professor with the School of Engineering Science. He was a Visiting Professor with the University of Victoria, Victoria, BC, Canada, from 1999 to 2000 . $\mathrm{He}$ has performed research in the areas of cellular radio networks and spreadspectrum systems since 1988. His current research interests include wideband, broadband, and ultrawideband CDMA for high-data-rate wireless multimedia, signal design and diversity techniques for CDMA/UWB and cross-layer design for CDMA/UWB systems.

Dr. Kim served as an Editor for the IEEE Journal on SELECTED AREAS In Communications: Wireless Communications Series and as a Division Editor for the Journal of Communications and Networks between 2001 and 2005. Currently, he serves as an Editor for Spread Spectrum Transmission and Access for the IEEE TRANSACTIONS ON COMMUNICATIONS and as an Editor for the IEEE TRANSACTIONS ON WIRELESS COMMUNICATIONS. 